\title{
A CNN Classification Model For Diagnosis Covid19
}

\author{
Ahmed Abdullah Farid ${ }^{1, *}$, Gamal Ibrahim Selim ${ }^{1,2}$, and Hatem Awad A. Khater ${ }^{3}$ \\ 1 Department of Computer Engineering, Faculty of Engineering \& Technology, Arab Academy for Science Technology and Maritime \\ Transport(AASTMT), Cairo, Egypt \\ 2 Professor Dr. Gamal Selim, (email: dgamal55@yahoo.com or dgamalselim@aast.edu ) \\ 3 Associate Professor Dr. Hatem Awad Aly Khater, Faculty of Engineering, Horus University in Egypt (email: hatem.a.khater@gmail.com) \\ * Corresponding author, (email: ahmed.abdullah.farid@gmail.com or portal.ahmed@ student.aast.edu )
}

\begin{abstract}
:
The paper demonstrates the analysis of Corona Virus Disease based on a CNN probabilistic model. It involves a technique for classification and prediction by recognizing typical and diagnostically most important CT images features relating to Corona Virus. The main contributions of the research include predicting the probability of recurrences in no recurrence (first time detection) cases at applying our proposed Convolution neural network structure. The Study is validated on 2002 chest $\mathrm{X}$-ray images with 60 confirmed positive covid 19 cases and ( 650 bacterial -412 viral -880 normal) $\mathrm{x}$-ray images. The proposed CNN compared with traditional classifiers with proposed CHFS feature extraction model. The experimental study has done with real data demonstrates the feasibility and potential of the proposed approach for the said cause. The result of proposed CNN structure has been successfully done to achieve $98.20 \%$ accuracy of covid 19 potential cases with comparable of traditional classifiers.
\end{abstract}

Keywords: convolution neural network, Composite Hybrid Feature Selection, Machine learning, Bacterial and Viral Pneumonia, CT-Image, MPEG7 edge histogram feature extraction, CNN.

\section{Introduction}

Data mining skills involved in biomedical sciences and investigate for providing prediction for help to identify the disease and classify it correctly[1]. Screening large numbers of reported cases for successful isolation and treatment is a priority to control the spread of Corona Virus Disease (COVID-19). Pathogenic laboratory testing is the scientific gold standard but, given significant falsenegative results, it is time-consuming. There is an urgent need for quick and accurate diagnosis methods to combat the disease. Based on COVID-19 radiographic improvements in CT scans, we tried to create a deep learning algorithm that could extract the graphical characteristics of COVID19 to provide a pre-pathogenic clinical diagnosis and thus save critical time for disease control[2-3]. Even before clinical symptoms emerge, computed tomography diagnose irregularities in patients with laboratory-confirmed coronavirus, according to a new case report[51-53]. It is yet another critical piece of evidence showing the central role of the modality in stopping the lethal epidemic. The case, reported in Clinical Imaging on February 22, reports that of a 61-year-old asymptomatic man admitted to a Chinese hospital 1,000 miles outside Wuhan after claiming close contact with an infected person[60]. In addition to identifying early abnormalities, CT showed a result that was not seen in any other COVID-19 instances. As well as those previously diagnosed with standard viral pneumonia and SARS, we obtained 250 CT photographs of pathogen-confirmed COVID-19 events from the Kaggle database web. Our proposed hybrid feature extraction of four filters ( MPEG-7 edge histogram filter with Gabor filter- pyramid of rotation-invariant local binary pattern histograms fuzzy 64-bin histogram ), which analyzes a low-level feature of an image can extract the features and provide a statistical hypothesis[40-50]. Our proposed model using composite hybrid attribute selection (CHFS) to achieve high accuracy in prediction and improve the feature extraction methods 
[2-15] with hybrid classification techniques for combine multi-classifiers to improving an in-depth investigation. Testing is employed to see which feature vectors/elements are most informative to differentiate different image classes. Also, using (CNN) for relatively little pre-processing compared to other image classification algorithms and traditional classifiers. The article planned as follows. The next section discusses the literature review of other authors who have used data mining and its relative of machine learning algorithm to analyze coronavirus. Section 3 describes the proposed technique used for feature extraction from CT images datasets with the CHFS model and four image filters. Section 4 describes the method used for the stack hybrid classification process and convolution neural network (CNN) in comparison with traditional classifiers, whereas section 5 describes the experiments and evaluation. Section 6 discusses the results. Finally, section 7 presents the paper summary and conclusions.

\section{Literature Review}

(Fei Shan) developed DL-based segmentation that uses the "VB-Net" neural network to segment CT scans of COVID-19 infection regions. The device is equipped using 249 patients with COVID19 and tested using 300 new patients with COVID-19. A human-in-the-loop (HITL) methodology is introduced to assist radiologists in developing automatic recording of each case to speed up the manual delineation of CT images for analysis. To assess DL-based system performance, the Dice similarity coefficient, volume differences, and infection percentage (POI) are calculated on the validation collection between automatic and manual segmentation checks. EOLBREAK (Xiaowei $\mathrm{Xu}$ ) observed that in the early stage of identification of viral RNA from sputum or nasopharyngeal swab, the real-time reverse transcription-polymerase chain reaction (RT-PCR) had a relatively low positive rate regarding evaluating COVID-19 (named World Health Organisation). COVID-19 computed tomography (CT) imagery manifestations had their characteristics, which vary from other forms of viral pneumonia, such as viral influenza-A pneumonia. Hospital doctors often lobby for another clear diagnostic criteria for this new type of pneumonia at the earliest possible opportunity. The accuracy of the chest CT (Yicheng Fang) was higher than that of RT-PCR (98\% vs. 71\% respectively, $\mathrm{p}<.001)$. The explanations for viral nucleic acid detection's low efficiency may include: 1) premature production of nucleic acid detection technology; 2) variability in the detection rate from different manufacturers; 3 ) weak viral load of patients; or 4) inadequate clinical sampling. (Xingzhi Xie, Zheng Zhong) observed that most instances have similar features on CT pictures, such as GGO or combined and merged GGOs. The peripheral spread of 2019-nCoV pneumonia is likely to occur with a longitudinal, multifocal lower lung involvement[6-8]. Given negative RT-PCR samples, CT characteristics of viral pneumonia may be highly suspect for 2019-nCoV infection in the case of regular clinical presentation, and exposure to other persons with 2019-nCoV. In these cases, it is essential to consider repeat swab testing and patient isolation.

Modern image edge detection algorithms include both first-order differential operators (i.e., operators Roberts, Prewitt, Sobel, and Canny) and second-order differential operators (i.e., operators Laplacian which LoG) and can extend to a wide variety of applications. Through integrating this with mathematical mechanics, Wang and Liu used the Roberts operator to identify vehicle image edges and distinguish vehicle license plate positions. While the detection mentioned above algorithms have the benefits of being simple and easy to implement and delivering excellent performance in real-time, they also have obvious shortcomings. The Roberts operator extracted image edge function is relatively rough and offers imprecise edge positions. The edge features which the Prewitt operator extracts have wide margins and many discontinuities. Likewise, the Sobel director does not provide precise picture edge coordinates. The Laplacian operator highly noised sensitive, and the LoG operator can not remove salt and pepper noise in an image.

\section{Proposed Work}


In the proposed work, the CT images dataset collected from the online access Kaggle benchmark dataset. The medical dataset contains several CT images for Sars and Covid-19 older adults - the proposed layout in Fig 1,2 .

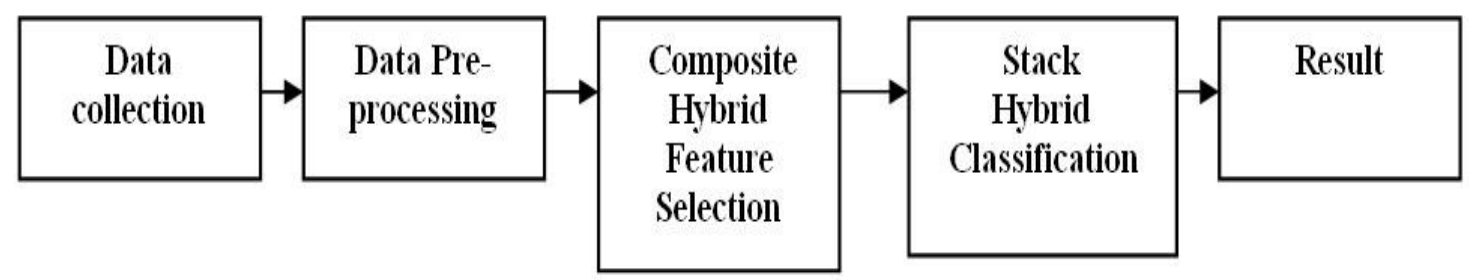

Fig. 1 The proposed layout for the clinical dataset



Fig. 2 The proposed feature extraction and classification for covid-19

The following steps explain the mechanism of the proposed work on CT images dataset :

\subsection{Data Collection}

The CT images dataset has Four classes of images both in training as well as the testing set containing a total of around 2002 chest x-ray images each segregated into the severity of bacterial and viral pneumonia and coronavirus (online access Kaggle benchmark dataset,2020):

i.Covid-19 iii.Viral

ii.Bacterial iv. Normal

\subsection{Data Pre-processing}

In the real world, data collected tend to be not wholly complete, noisy and conflicting, detection missing of data, data irregularity, prevent the errors, and decrease the data to be analyzed would lead to massive payouts for decision making (H. Witten \& Eibe Frank ,2008). 
For machine learning and analytics, the selection of features is the process of selecting a subset of specific features (variables, predictors) for use in model building. Component selection methods used for many reasons(H. Witten \& Eibe Frank ,2008):

i.simplification of the models to make them easier for researchers/users to understand ii.shorter training periods

iii.preventing the curse of dimensionality

iv.improved generalization by minimizing overfitting

\section{3.proposed Hybrid Feature Selection Approach on CT-Images}

There is a variability of approaches used to obtain images. Some of these are the mean and difference dependent on the bi-orthogonal wavelet filter, image retrieval based on shape. Edges are an essential image feature that is present between an objective and a background and between two targets, two zones, or two primitives. Much of the details for a picture borne in the margins. A picture edge is usually a group of pixels for which the gray-level values demonstrate a shift in phase(Jianfang Cao,2018)[12-22].

\subsubsection{MPEG7 Histogram Filter}

The histogram is the form most widely used to describe an image's composition of any global function. Translation and rotation of the picture invariant, a normalization of the histogram corresponds to the invariance of the distance. According to the edge histogram description in MPEG7 , an extra histogram bin may easily be created from the local 5-bin edge histogram of each $4 * 4$ subimage. A statistical hypothesis testing is employed to see which feature vectors/elements are most informative to differentiate different image classes. The histogram is very useful for indexing and extracting pictures using the above property.

\subsubsection{Gabor Image Filter}

Gabor filter used for texture analysis, which implies that it mainly analyzes if there is some different frequency information in the picture in specific directions across the point or area of analysis in a regional region. It can define by a sinusoidal wave (a plane wave for 2D Gabor filters) multiplied by a Gaussian function. A set of Gabor filters with different frequencies and orientations may assist in extracting useful features from an image. In the discrete domain, the two-dimensional Gabor filters are given by(2)-(3),

$$
\begin{aligned}
& G_{c}[i, j]=B e^{-\frac{\left(i^{2}+j^{2}\right)}{2 \sigma^{2}}} \cos (2 \pi f(i \cos \theta+j \sin \theta)) \\
& G_{s}[i, j]=C e^{-\frac{\left(i^{2}+j^{2}\right)}{2 \sigma^{2}}} \sin (2 \pi f(i \cos \theta+j \sin \theta))
\end{aligned}
$$

\subsubsection{Pyramid of Rotation-Invariant Local Binary Pattern Histograms Image Filter}

The local binary sequence (LBP) commonly used in the classification of textures. The modern LBP methods define only micro-texture picture structures, such as edges, corners, points, although many of them show excellent texture classification efficiency. This situation could still not be changed, although the technique of multi-resolution research used in local binary pattern methods(Ojala et al., 2002). The texture operator LBP has become a simplified approach in different applications. These can describe as a unifying solution to historically different computational and structural texture research frameworks.

To the LBP operator, the following terminology used: LBPP, Ru2. The subscript represents a neighborhood using the operator $(\mathrm{P}, \mathrm{R})$. Superscript $\mathrm{u} 2$ indicates that only standard patterns used and 
the other patterns marked with a single label. After obtaining the LBP-labeled picture fl(x,y), the LBP histogram can identify as

$$
H i=\sum x, y I\{f l(x, y)=i\}, i=0, \ldots, n-1,
$$

$\mathrm{N}$ is the number of different LBP operators labels, and $\mathrm{I}\{\mathrm{A}\}$ is 1 if $\mathrm{A}$ is valid and 0 if $\mathrm{A}$ is false. When the picture patches with different sizes applied to the histograms, the histograms must standardized to achieve a coherent description:

$$
N i=H i \sum n-1 j=0 H j
$$

\subsubsection{Fuzzy 64-bin Histogram Image Filter}

The Fuzzy 64-bin focused on color vision is usually not typically shown in RGB. The better model of HVS is the so-called opposing color type. The competing color space has three component fuzzy 64-bin bases on color expectations typically not better represented in RGB. (C. A. Bouman,2007):

- O1 is a luminance component

- $\mathrm{O} 2$ is the red-green channel

- $\mathrm{O} 2=\mathrm{G}-\mathrm{R}$

- O3 is the blue-yellow channel

- $\mathrm{O} 3=\mathrm{B}-\mathrm{Y}=\mathrm{B}-(\mathrm{R}+\mathrm{G})$

Consequences of Opponent Channel CSF Luminance channel is Bandpass function

- Wide bandwidth $\Rightarrow$ high spatial resolution.

- Low-frequency cut-off is $\Rightarrow$ insensitive to the average luminance level. Chrominance channels are

- Lowpass function

- Lower bandwidth $\Rightarrow$ low spatial resolution.

- Low pass $\Rightarrow$ sensitive to absolute chromaticity (hue and saturation). (C. A. Bouman,2007)

\subsubsection{Applying Hybrid feature selection architecture model on CT-images}

The author combines four filters to feature extraction process considered for the optimal selection feature set from CT- images, as shown in fig 3 .

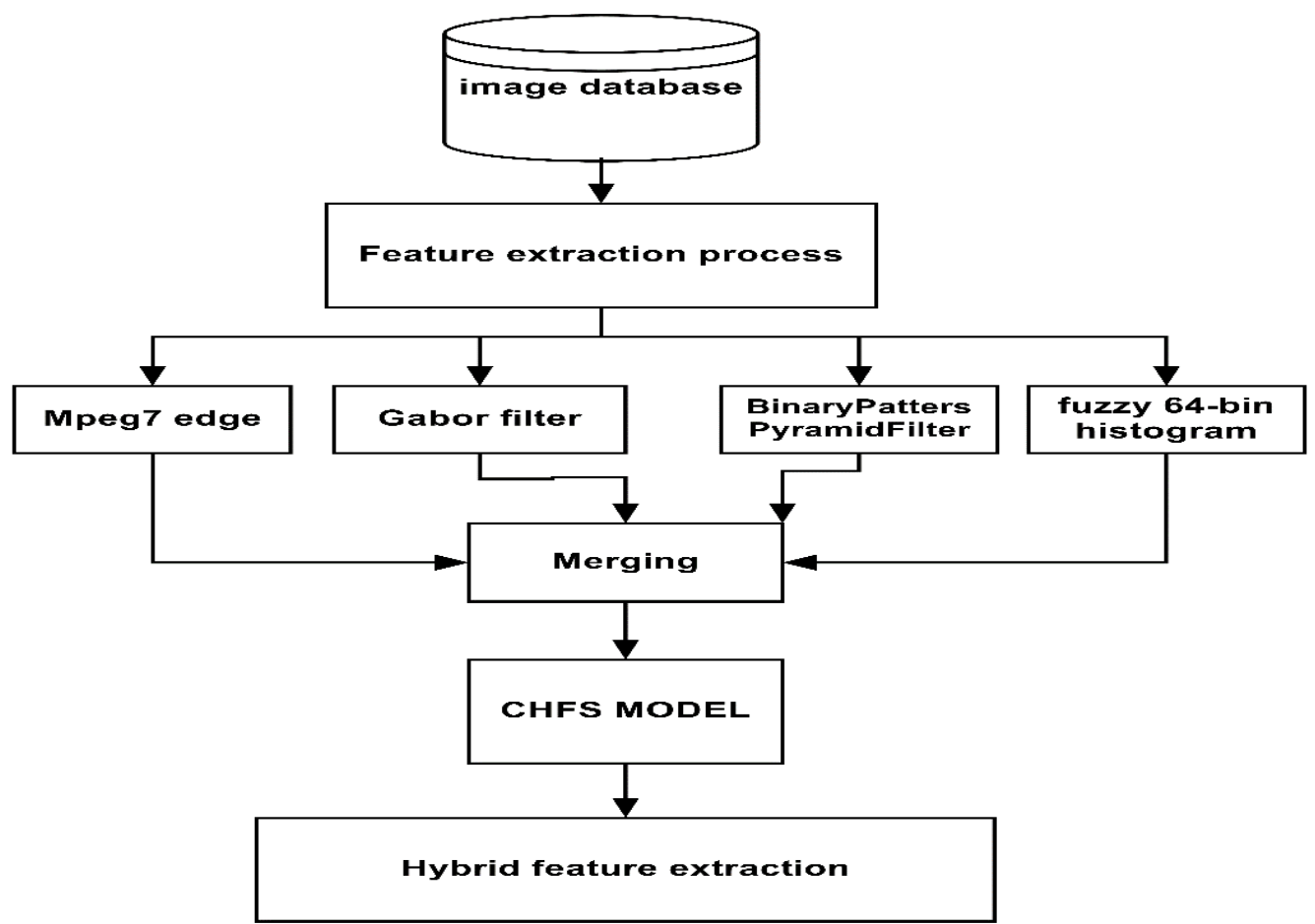

Fig. 3 The proposed hybrid feature selection of CT-images architecture 


\subsubsection{Proposed Composite Hybrid Feature Selection Model (CHFS)}

Feature selection is the approach of taking a subset of relevant features for use in model construction(Chen \& ...\& F. Li,2010), and combines the advantages of three feature selection approaches (Filter (IG,GR)-Wrapper( improved (Genetic Algorithm)) with Embedded(C4.5)).

\subsubsection{Composed Hybrid feature selection architecture}

The author combine of three feature extraction technique considered for the optimal selection feature set, and this method is information gain (IG) - gain ratio (GR) and Optimized Genetic Algorithm(Huang \& C.,2012) (Yanan Mao \& Dingyuan Fan,2016)[18-26]. As shown in fig 4.

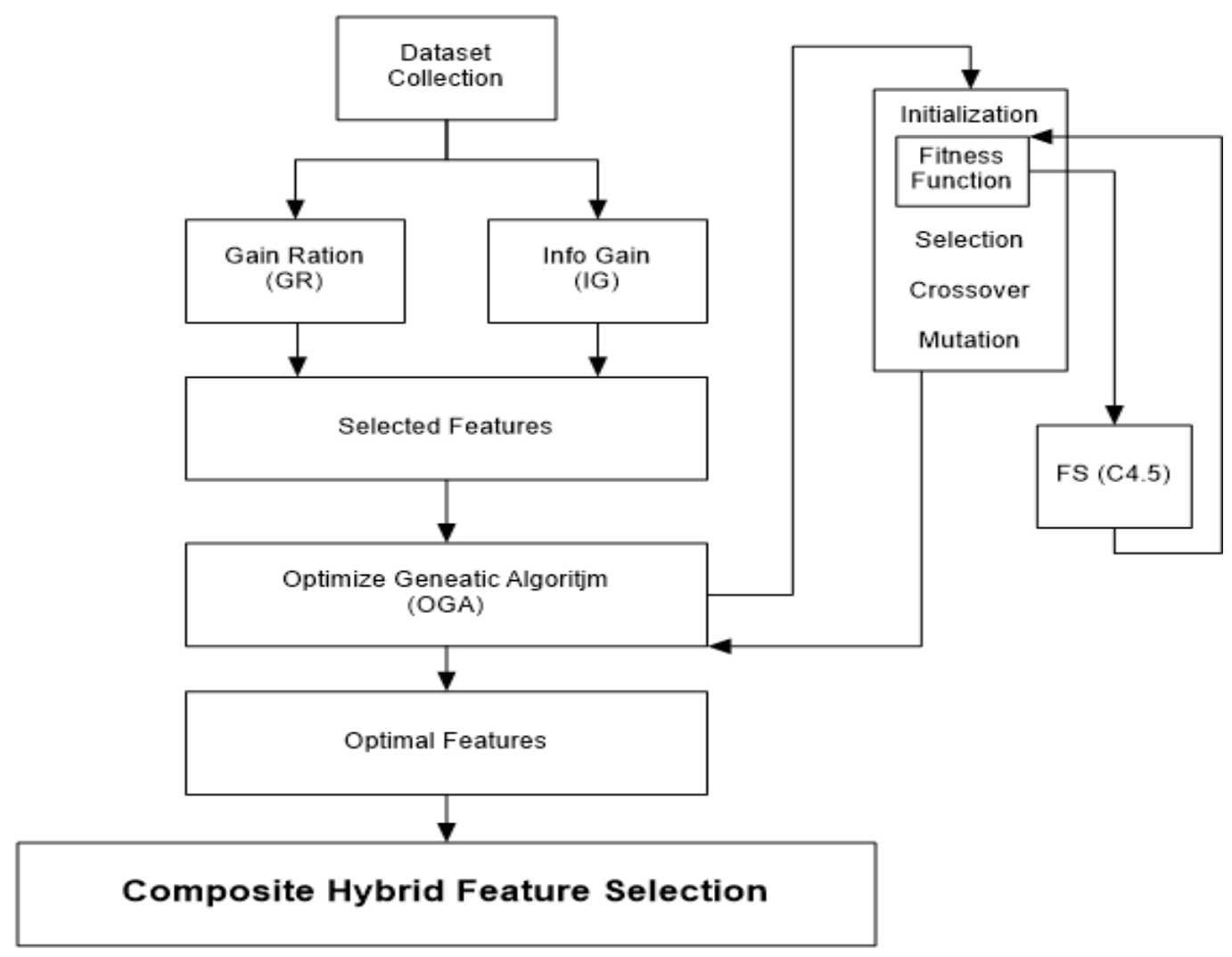

Fig.4 The proposed composite hybrid feature selection architecture

In Figure 4, The superior ranked features taken as the optimized subset, the threshold established the size of the resumption feature subset and amount of attributes in the dataset that appear in the range(44 50) (Vemuri \& et al.Jack \& C. R.,2008) (Devi \&Cuckoo ,2013)[15-22].

\subsubsection{Information Gain feature selection}

The calculation of the information gain for only one attribute according to the algorithm below (Aouatif Amine \& ...\& Rziza Driss ,2011)[28-32]: This gain measure gives the effect of the features, and the following algorithm selects features that are larger than the threshold.

\subsubsection{Gain Ratio Feature Selection}


A decision tree can be a simple form when non-terminal nodes perform tests on many attributes to the effect of decision outcomes (J.R. Quinlan,1986)[38]. The gain ratio is

$$
\operatorname{GainRatio}(A)=\operatorname{Gain} \frac{A}{-} \operatorname{QplitInfoA}(Q)
$$

\subsubsection{Optimized Genetic Algorithm (OGA)}

The authors propose a method to modify a general genetic algorithm to evaluates specified attributes on training data or a separate testing set and uses a decision tree (J.R. Quinlan,1986) [3238]to estimate the 'merit' of a set of attributes to produce an optimized feature subset with genetic search elevation strategy to recognize the features. All feature selection technique should use an evaluation function together with a search strategy to achieve the optimal feature set(Huang \& C.,2012)[44].

It is unable to be realized to search all subsets to find out an optimal subset and need much effort to indicate whether a particular feature is present or not in the chromosome, one, and zero used. One in a gene position refers to feature and zero to absent(Yanan Mao \& Dingyuan Fan,2016).The number of features and what are the features that are to be present in a chromosome are guided by information gain (IG) and gain ratio (GR).

The initial population created using input values of IG and GR of the values present in the chromosome. After Generated the population, the individuals evaluated using a fitness function. There is no general approach to find the fitness function for a genetic algorithm. It is a heuristic approach and depends on the used application. So the authors nominate a C4.5 classifier to be used as a fitness function because $\mathrm{C} 4.5$ has some utility of handling both continuous and discrete attributes and training data with missing attribute values, pruning trees after creation - C4.5 goes back through the tree once it has been created and try to eject branches that do not help by replacing them with leaf nodes (Dash \& H. Liu, 1997) . (J.R. Quinlan,1986). The following algorithm selects a feature from the set of features that are gained by OGA, gain ratio, and Information gain, as shown in fig 5.

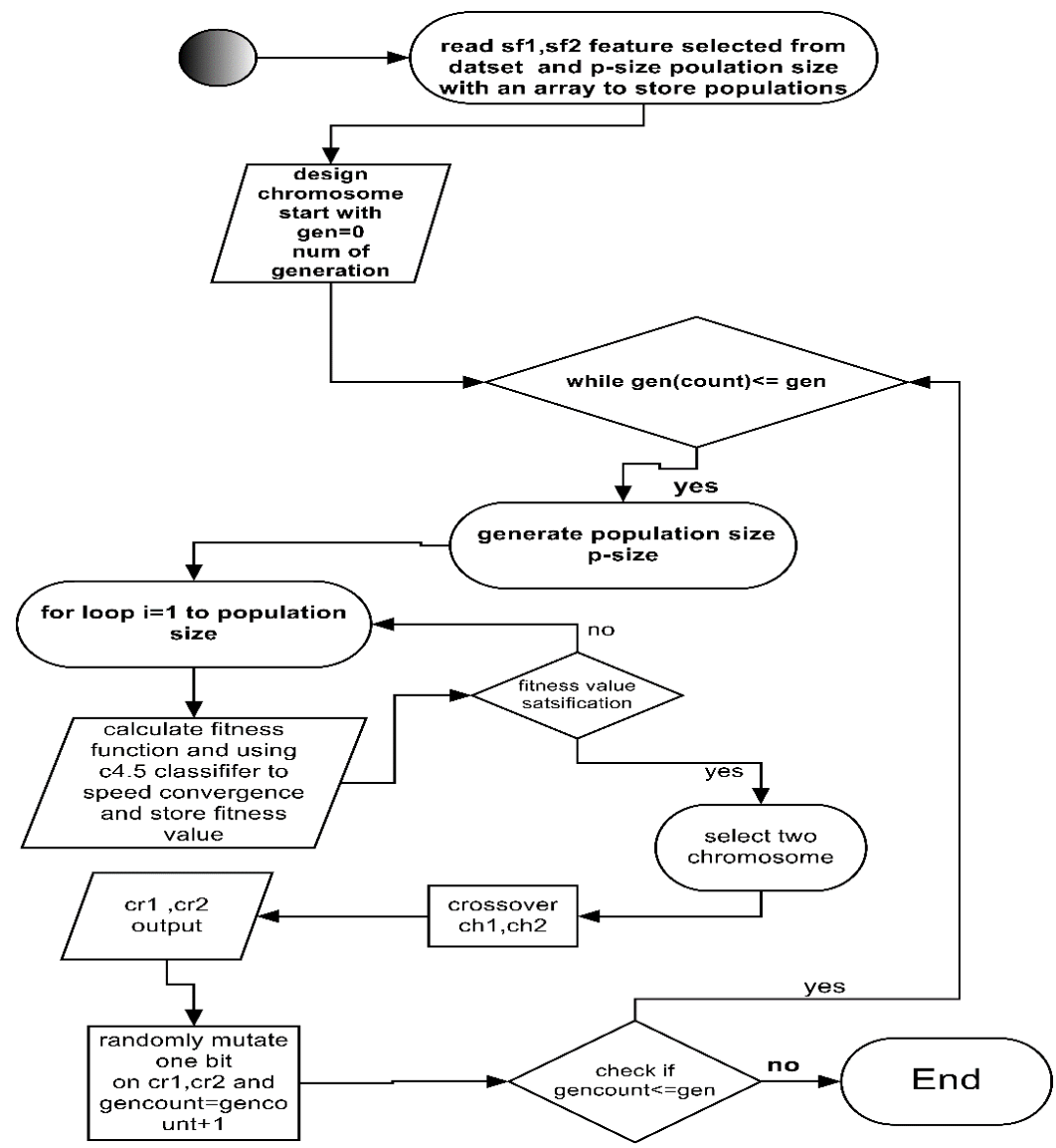

Fig. 5 Proposed optimized genetic algorithm with the c4.5 decision tree 


\subsection{Proposed Stack Hybrid Classification Model}

A weka software tool (Weka online open-source accessed,2018)[16] shows the list of blackbox classifiers. These algorithms, in general, are used to classify the medical dataset.

\subsubsection{Two learning evaluators can be used to evaluate the dataset}

- Training set: the classifier separates a dataset to test and training data.

- Cross-validation: in case of 10 fold cross-validation (Divya Jain, Vijendra Singh,2018).

\subsubsection{Metrics used in health check systems for evaluation}

The different performance metrics generally used to explore the performance of the different models like sensitivity, accuracy, precision, and f-measure (Divya Jain \& Vijendra ,2017) . Accuracy: can be calculated by divide number of accurate predictions by the total number of all predications (Klöppel \& ...\&Frackowiak \& R. S,2008)

Inside equation

$$
\text { Accuracy }=\frac{T N+T P}{f P+t P+f N+t N}
$$

$(\mathrm{FN}=$ False Negative, $\mathrm{FP}=$ False Positive, $\mathrm{TN}=$ True Negative, $\mathrm{TP}=$ True Positive $)$

\section{Experimental Results and Evaluation}

The CSV file of 2002 x-ray Dataset to patients from Kaggle.com loaded to the weka tool. All experiments evaluated by the receiver operating characteristic (ROC) curve, accuracy, F-measure.

\subsection{Result from proposed (CHFS) feature selection model}

The CT-images dataset collected from Kaggle database website with a total of 51 images each segregated into the severity of COVID-19[74-76], first we apply four image filter mention in the proposed architecture above in fig 6 on x-ray images to extract the mathematical data analysis relation to applying the CHFS model and the result of feature extracted shown below in table1.

Table 1:Number of feature extraction from hybrid-image-filter-CHFS feature selection model

\begin{tabular}{|c|c|c|}
\hline & Method & $\begin{array}{c}\text { No.of } \\
\text { Features }\end{array}$ \\
\hline MPEG-7 & Image filter & 82 \\
\hline Gabor & Image filter & 142 \\
\hline Binary patterns pyramid & Image filter & 898 \\
\hline fuzzy 64 bin histogram & Image filter & 1473 \\
\hline IG, GR & Filter & 2100 \\
\hline GA & Wrapper & 269 \\
\hline $\begin{array}{c}\text { Optimized GA Fitness } \\
\text { Fun(C4.5)(OGA) }\end{array}$ & Embedded & 165 \\
\hline
\end{tabular}

Besides using four image filters to extract the features from x-ray images, which extract 82 features from MPEG-7 filter and 142 features after applying Gabor filter with 898 features from binary patterns pyramid with final applying of fuzzy 64-bin histogram to produce total 1473 features from CT-images dataset. Besides, applying our proposed CHFS with optimization of genetic algorithm to get the last 165 sensitive features[76]. 
If 10- fold cross-validation applied. For summarized results in the table (2) from the weka software of the analytics dataset, we used the (ROC) curve to evaluate the output result from our proposed architecture model, as shown in fig 8.

Table 2: Experimental result of Accuracy pre-post CHFS model with various classifiers on CT-images images

\begin{tabular}{|c|c|c|}
\hline Classifier & $\begin{array}{c}\text { Pre- (CHFS) Feature } \\
\text { Selection Accuracy } \\
\text { By using four image } \\
\text { filter with 1473 } \\
\text { feature }\end{array}$ & $\begin{array}{c}\text { Post - } \\
\text { (CHFS) } \\
\text { Feature } \\
\text { Selection } \\
\text { Accuracy }\end{array}$ \\
\hline SVM & $77.31 \%$ & $71.36 \%$ \\
\hline Naïve Bayes & $66.41 \%$ & $68.96 \%$ \\
\hline JRIP & $68.86 \%$ & $67.91 \%$ \\
\hline Random Forest & $76.71 \%$ & $77.36 \%$ \\
\hline & & \\
\hline
\end{tabular}

The overall improvement in our experiment on traditional classifiers produce $13.87 \%$ after using our proposed feature extraction of traditional model from CT-images[77]. The figure below shows it summarize for all results in table 6 .

\begin{tabular}{|lllllllll|}
\hline TP Rate & FP Rate & Precision & Recal1 & F-Measure & MCC & ROC Area & PRC Area & Class \\
0.928 & 0.359 & 0.553 & 0.928 & 0.693 & 0.534 & 0.784 & 0.537 & BacterialPneumonia \\
0.017 & 0.000 & 1.000 & 0.017 & 0.033 & 0.127 & 0.508 & 0.046 & Covid \\
0.861 & 0.045 & 0.937 & 0.861 & 0.898 & 0.825 & 0.908 & 0.868 & Normal \\
0.163 & 0.023 & 0.650 & 0.163 & 0.260 & 0.256 & 0.570 & 0.278 & ViralPneumonia \\
0.714 & 0.141 & 0.755 & 0.714 & 0.674 & 0.593 & 0.786 & 0.614 & \\
\hline
\end{tabular}

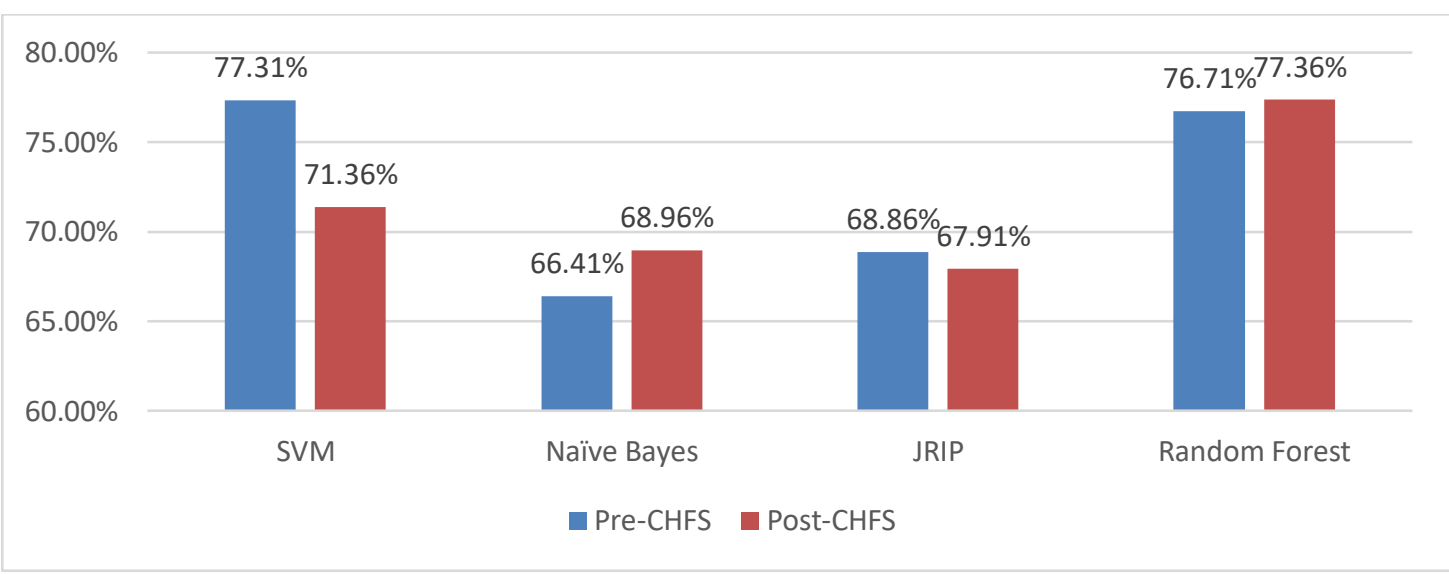

Fig.8 Summarized accuracy results of pre-post (CHFS) feature selection

\subsection{Applying proposed Convolution neural network (CNN) on covid-19-normal-bacterial-viral $x$ - ray images}

The author's aims to make a comparison between the proposed traditional model and proposed modifying convolution neural network $(\mathrm{CNN})$ model structure, We tested a set of x-ray-images of COVID-19--normal-bacterial-viral x-ray images from the kaggle.com benchmark web of dataset science, as shown in below figure8, to perform the accuracy of early-screen diagnosis by the convolution neural network. 


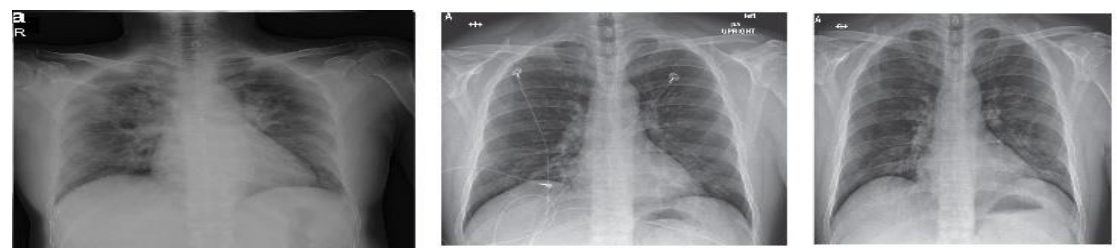

Fig. 9 the types of covid19-ct-images

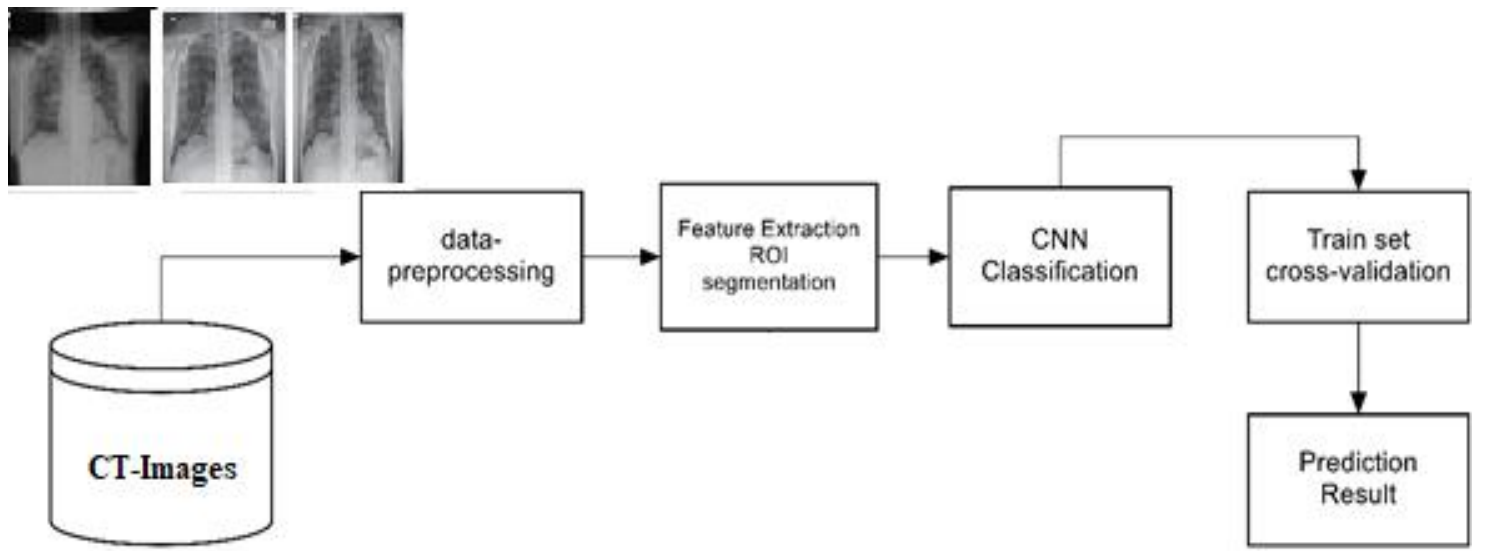

Fig. 10 (a)shows the prediction model for CNN CT-images
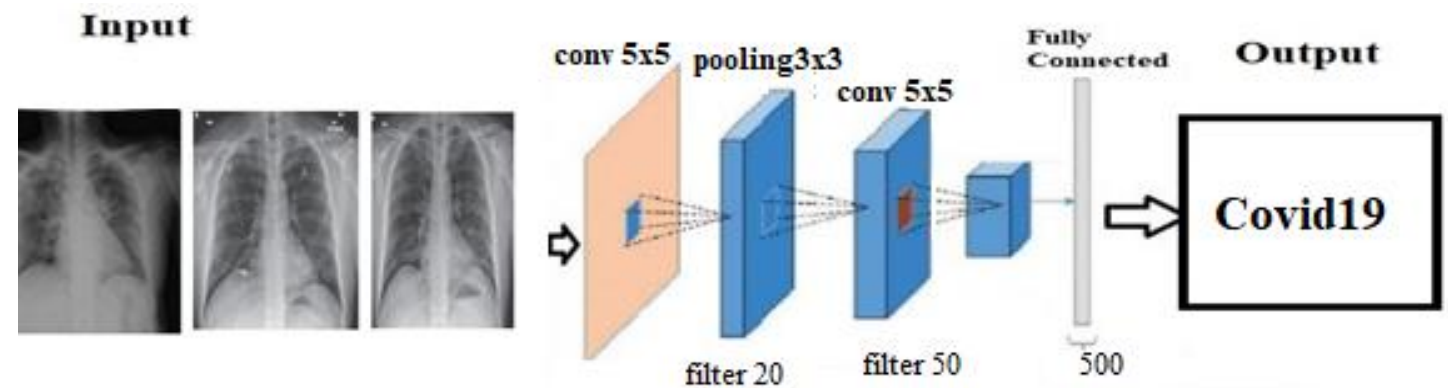

Fig. $10(\mathrm{~b})$ shows the structure model for proposed CNN CT-images

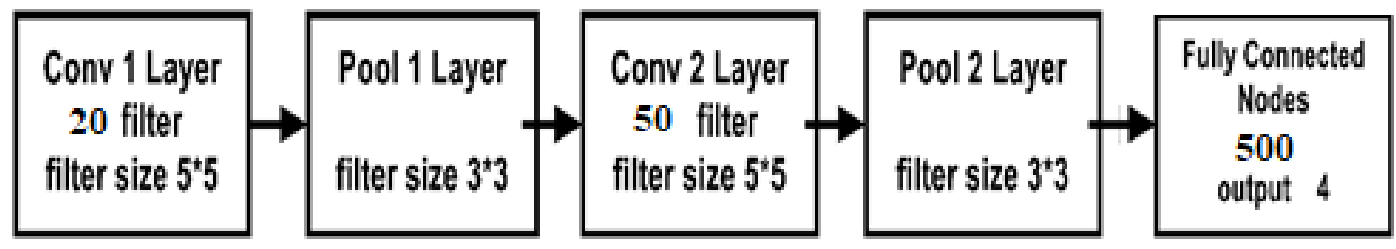

Fig. 10 (c)shows the cnn network structure

In the below figure 11, we propose the result of ct-images-CNN classification on Kaggle 2002 CT-images dataset by using the convolution neural network in weka software data mining, the result evaluated by ROC curve - f-measure 


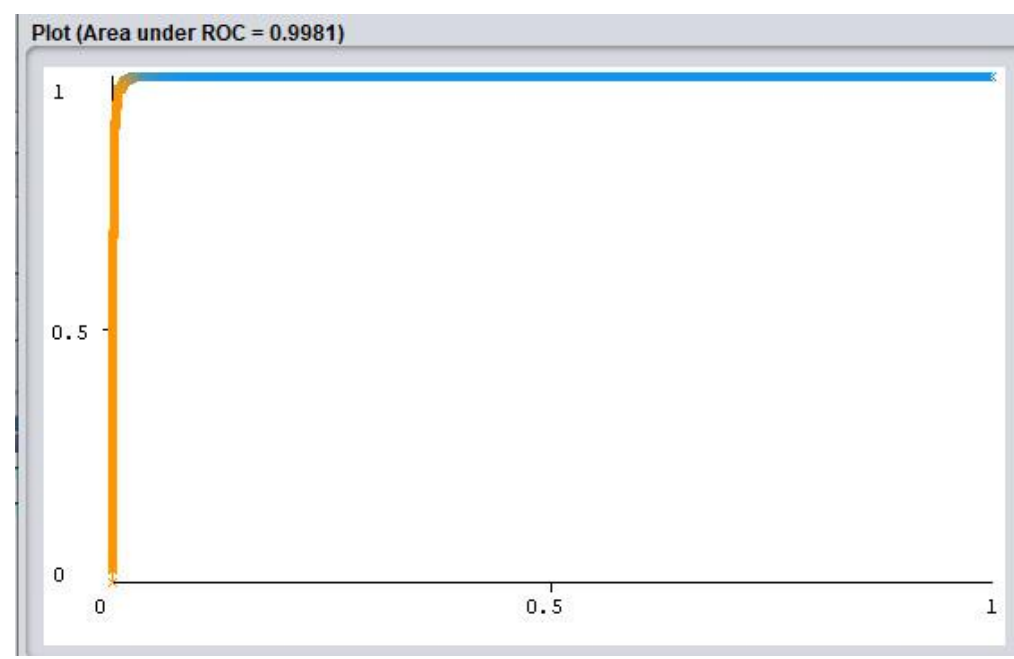

$===$ Confusion Matrix $===$

\begin{tabular}{|c|c|c|c|c|}
\hline$a$ & b & C & d & $<--$ classified as \\
\hline 647 & 0 & 0 & 3 & $a=$ BacterialPneumonia \\
\hline 0 & 60 & 0 & 0 & $\mathbf{b}=$ COVID \\
\hline 0 & 0 & 880 & 0 & $c=$ Normal \\
\hline 32 & 0 & 1 & 379 & d = ViralPneumonia \\
\hline
\end{tabular}

Fig.11 ROC \& confusion matrix curve of CT-images category and confusion matrix of CNN classification

And in the below figure 12 shown the error curve of CNN classification.

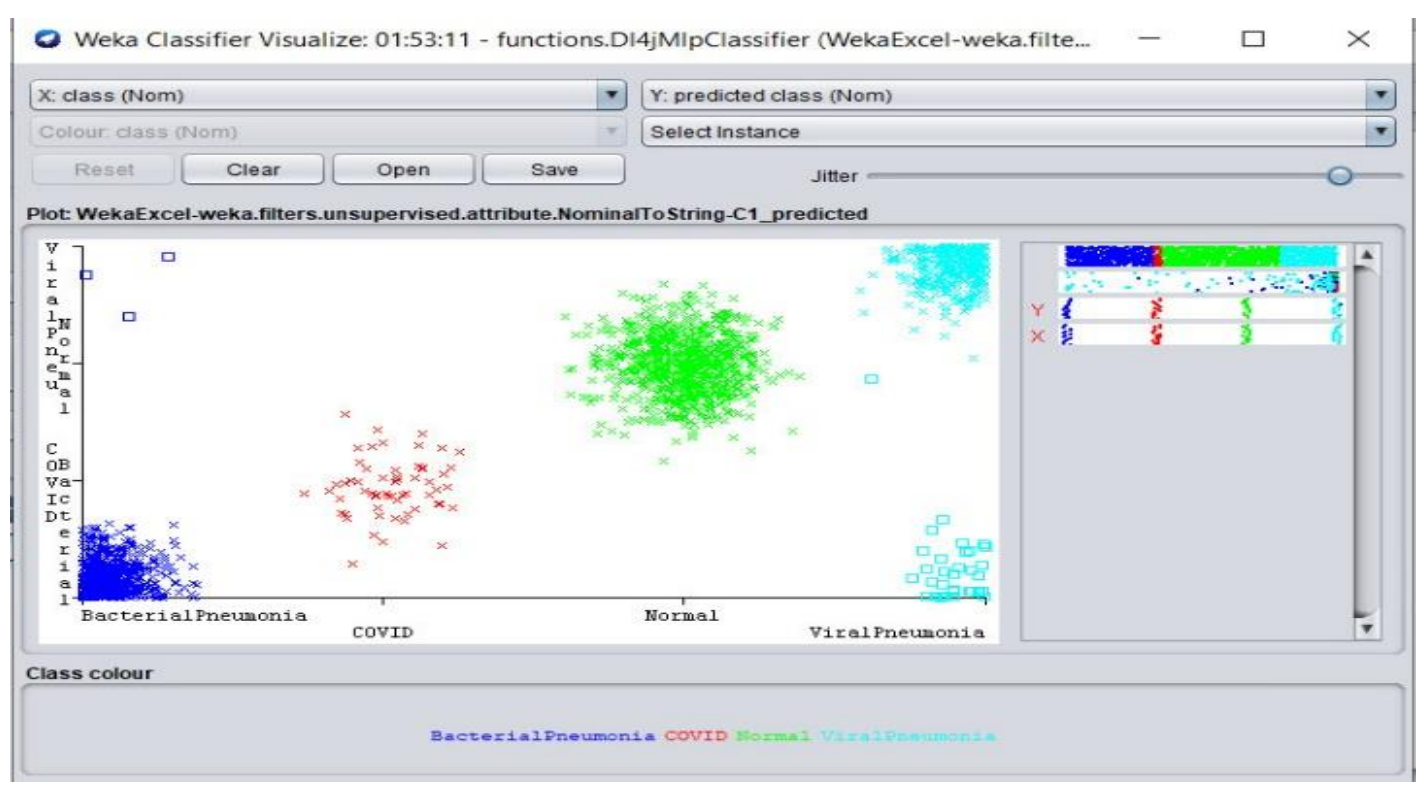

Fig.12 shown the error curve of CNN classification process 
Table3: The details of result from CNN-Model

\begin{tabular}{|c|c|c|c|}
\hline F-measure & TPR & ROC & PRC \\
\hline $98.2 \%$ & $98.2 \%$ & $99.9 \%$ & $99.7 \%$ \\
\hline
\end{tabular}

The classification accuracy of the convolution neural network (CNN) was $98.20 \%$ and f-measure $98.2 \%$, which considered better than the traditional classififers accuracy .

\begin{tabular}{|c|c|c|c|c|c|c|c|c|c|}
\hline \multicolumn{3}{|c|}{ Correctly Classified Instances } & \multicolumn{2}{|l|}{1966} & \multicolumn{2}{|c|}{$98.2018 \%$} & & & \\
\hline \multirow{2}{*}{\multicolumn{3}{|c|}{$\begin{array}{l}\text { Incorrectly Classified Instances } \\
\text { Kappa statistic }\end{array}$}} & \multicolumn{2}{|l|}{36} & \multicolumn{2}{|c|}{$1.7982 \%$} & & & \\
\hline & & & \multicolumn{2}{|c|}{0.9726} & & & & & \\
\hline \multicolumn{3}{|c|}{ Mean absolute error } & \multicolumn{2}{|c|}{0.0111} & & & & & \\
\hline \multicolumn{3}{|c|}{ Root mean squared error } & \multicolumn{2}{|c|}{0.0826} & & & & & \\
\hline \multicolumn{3}{|c|}{ Relative absolute error } & \multicolumn{2}{|c|}{$3.3852 \%$} & & & & & \\
\hline \multicolumn{3}{|c|}{ Root relative squared error } & \multicolumn{2}{|c|}{$20.3626 \%$} & & & & & \\
\hline \multicolumn{3}{|c|}{ Total Number of Instances } & \multicolumn{2}{|c|}{2002} & & & & & \\
\hline \multicolumn{10}{|c|}{$===$ Detailed Accuracy By Class == } \\
\hline & TP Rate & FP Rate & Precision & Recall & F-Measure & MCC & ROC Area & PRC Area & Class \\
\hline & 0.995 & 0.024 & 0.953 & 0.995 & 0.974 & 0.961 & 0.998 & 0.994 & BacterialPneumonia \\
\hline & 1.000 & 0.000 & 1.000 & 1.000 & 1.000 & 1.000 & 1.000 & 1.000 & COVID \\
\hline & 1.000 & 0.001 & 0.999 & 1.000 & 0.999 & 0.999 & 1.000 & 1.000 & Normal \\
\hline & 0.920 & 0.002 & 0.992 & 0.920 & 0.955 & 0.945 & 0.997 & 0.993 & ViralPneumonia \\
\hline Weighted Avg. & 0.982 & 0.008 & 0.983 & 0.982 & 0.982 & 0.976 & 0.999 & 0.997 & \\
\hline
\end{tabular}

Fig12. The result of four class of Kaggle dataset after applying proposed CNN model

\section{Discussion}

The authors compare the results of the proposed feature extraction model on covid19Bacterial -viral-normal x-ray -images data in different cases pre-post feature extraction model and the result compared with proposed CNN structure model. These comparisons, according to our proposed model presented in this study, were reduced a false negative rate and showed a relatively high overall accuracy with more accurate results. The best result extracted from our proposed CNN model was achieved accuracy of $98.20 \%$ compared to the output result of traditional classifiers achieved of accuracy of $77.36 \%$. This result make convolution neural network and deep learning plays a vital role in diagnosing a serious global epidemic that has no cure.

\section{Conclusion}

In this work, the author aims to early diagnosis of covid19 by using a benchmark x-rayImages dataset on our proposed combination of four image filters with composed hybrid feature selection (CHFS) model. That combines the advantages of three filter feature selection approaches and optimizes the Genetic Algorithm (OGA) by improving the initial population generating and genetic operators. Using the results of filter approaches as some prior information with using the $\mathrm{J} 48$ decision tree classifier as a fitness function instead of probability and random selection to speed up convergence and select the best features with compare of modifying a novel CNN structure which contain $2 \mathrm{CNN}$ of $(20-50)$ filters to obtain the best way of diagnosis,. The proposed CNN model shows better than the traditional classification approaches for optimum feature selection and improvement of the classification process and effectively reduced the false-negative rate with high accuracy with $98.20 \%$ compared to $77.31 \%$ of usage traditional classififers individually after proposed chfs feature selection model which is considerably better than the previous state-of-the-art result. The results of the proposed CNN model show an accurate classify of $\mathrm{x}$ ray-Images of COVID19 between viral and bacterial pneumonia. 


\section{References}

[1]Kalló, Gergö, Miklós Emri, et al. "Changes in the Chemical Barrier Composition of Tears in Alzheimer's Disease Reveal Potential Tear Diagnostic Biomarkers." PLoS One, vol. 11, no. 6, Public Library of Science, June 2016, p. e0158000.

[2] J. Escudero, et al. Machine learning-based method for personalized and cost-effective detection of Alzheimer's disease. IEEE transactions on biomedical engineering 2013; 60(1): 164-168.

[3] Jyoti Soni et al. "Predictive Data Mining for medical diagnosis: An Overview of Heart Disease Prediction"International Journal of Computer Applications (0975 -8887), Volume 17, No. 8, March 2011."

[4] Williams, Jennifer A., Alyssa Weakley, Diane J. Cook, and Maureen Schmitter-Edgecombe. (2013) "ML techniques for diagnostic differentiation of mild cognitive impairment." In Workshops at the Twenty-Seventh AAAI Conference on Artificial Intelligence: 71-76...

[5] Chi, C. L., Oh, W., \&Borson, S. (2015) "Feasibility Study of a Machine Learning Approach to Predict Dementia Progression," In Healthcare Informatics (ICHI), International Conference: 450450

[6] Chyzhyk, D., \&Savio, A. (2010) "Feature extraction from structural MRI: data from OASIS database," University of The Basque Country, Internal research publication.

[7] S. R Bhagya Shree and Dr. H. S. Sheshadri "An initial investigation in the diagnosis of Alzheimer's disease using various classification techniques" IEEE ICCIC, 2014.

[8] Tina R. Patil and Mrs. S. S. Sherekar "Performance Analysis of Naive Bayes and J48 Classification Algorithm for Data Classification" International Journal of Computer Science and Applications Vol. 6, No.2, Apr 2013

[9] Jihad Ali et al. "Random Forests and Decision Trees" IJCSI International Journal of Computer IS, Vo. 9, Issue 5, No 3, September 2012

[10] Vemuri, P., Gunter, J. L., Senjem, M. L., Whitwell, J. L., Kantarci, K., Knopman, D. S., ... \& Jack, C. R. (2008) "Alzheimer's disease diagnosis individual subjects using structural MR images: validation studies," Neuroimage, 39(3): 1186-1197.

[11] Datta, P., and Shankle, W. R., \&Pazzani, M. (1996) “Applying ML to an Alzheimer's database," In Conference proceedings of the AAAI symposium: 25-27.

[12] Klöppel, S., Stonnington, C. M., Chu, C., Draganski, B., Scahill, R. I., Rohrer, J. D., ... \&Frackowiak, R. S (2008)“Automatic classification of MR scans in Alzheimer's disease.” Brain, 131(3): 681-689.

[13] So, A., Hooshyar, D., Park, K. W., \& Lim, H. S. (2008) "Early Diagnosis of Dementia from Clinical Data by Machine Learning Techniques," Applied Sciences, 7(7): 651.

[14] Ramirez, J., Górriz, J. M., Salas-Gonzalez, D., Romero, A., López, M., Álvarez, I., \& GómezRío, M. (2013) "Computer-aided diagnosis of Alzheimer's type dementia combining support vector machines and discriminant set of features." Information Sciences, 237: 59-72.

[15] Sheshadri, H. S., Shree, S. B., \& Krishna, M. (2015) "Diagnosis of Alzheimer's Disease Employing Neuropsychological and Classification Techniques," In IT Convergence and Security (ICITCS), 2015 5th International Conference: 1-6.

[16]Alzehimer's clinical medical dataset available online at https://www.oasis-brains.org/\#Data (accessed on 2 Jan 2019) OASIS-3: Principal Investigators: T. Benzinger, D. Marcus, J. Morris; NIH P50AG00561, P30NS09857781, P01AG026276, P01AG003991, R01AG043434, UL1TR000448, R01EB009352. AV-45 doses provided by Avid Radiopharmaceuticals, a wholly-owned subsidiary of Eli Lilly OASIS-3: https://doi.org/10.1101/2019.12.13.19014902

[17] Data mining: Practical machine learning tools and techniques by Ian H Witten and Eibe Frank, published by Elsevier, second edition 2008.

[18]Aouatif Amine Ali Elakad Mohammed Rziza Driss "GA-SVM and Mutual Information based Frequency Feature Selection for Face Recognition" GSCM-LRIT, Faculty of Sciences, Mohammed V University, B.P. 1014 Rabat, Morocco(2011).

[19] J.R. Quinlan, Induction of Decision Trees, Machine Learning 1: pp.81-106, Kluwer Academic Publishers, Boston, (1986).

[20] Leo Breiman. "Stacked Regressions" Machine Learning, 24, 49-64 (1996) 
[21] Duarte Ferreira et al. "Applying data mining techniques to improve diagnosis in neonatal jaundice" BMC Medical Informatics and Decision Making, 0-5, 2012

[22] Jiawei Han, Micheline Kamber, Jian Pei "Data Mining: Concepts and Techniques" published by Elsevier, Third edition, 2012

[23] Anil Rajput, Ramesh Prasad Agarwal, Meghna Dubey S.P. Saxena, and Manmohan Raghuvanshi" J48 and JRIP Rules for EGovernance Data" International Journal of Computer Science and Security (IJCSS), Volume (5): Issue (2), Pp 201, 2011.

[24]Guyon I, Elisseeff A An introduction to variable and feature selection J Mach Learn Res 2003; 3(Mar):1157-82.

[25] Feature selection and classification systems for chronic disease prediction: A review Divya Jain, Vijendra SinghThe NorthCap University, Gurugram 12,2017, India, [online ] http://creativecommons.org/licenses/by-nc-nd/4.0

[26] Huang, C., A hybrid stock selection model using genetic algorithms and support vector regression. Applied Soft Computing, 2012. 12(2): p.807-818

[27] K. Tejeswinee, S.G. Jacob. Binary classification of Cognitive Disorders: Investigation on the Effects of Protein Sequence Properties in Alzheimer's and Parkinson's disease. IAENG-IMECS 2017: 166-170

[28] Joliffe IT. Principal component analysis, series: Springer series in statistics. 2. Springer; NY: 2002

[29]O. Elsayed, K. Mahar, M. Kholief and H. A. Khater, "Automatic detection of the pulmonary nodules from CT images," 2015 SAI Intelligent Systems Conference (IntelliSys), IEEE, London, pp.742-746, 2015, DOI: 10.1109/IntelliSys.2015.7361223.

[30]Yasser Fouad, Hatem Khater, Mostafa Setta, Ashraf Alsaid, "proposed Approach for Automatic Underwater Object Classification," International Journal of Research and Surveys, ICIC -EL, Volume 12, No. 12, pp. 1202-1212, 2018, DOI:10.24507/icicel.12.12.1205.

[31] The national center for health statistics report for 2019 (https://www.cdc.gov/nchs/fastats/leading-causes-of-death.htm).

[32] Weka open source software for data mining .availble online (https://www.cs.wakito.ac.nz/ ) accessed 26 Dec 2019.

[33] Chen, F. and F. Li, A combination of feature selection approach with SVM in credit scoring. Expert Systems with Applications, 2010. 37(7): p. 4902-4909.

[34] Chen, Y. -W., and Lin, C. -J., Combining SVMs with various feature selection Strategies, Available from http://www.csie.ntu.edu.tw/ cjlin/papers/features.pdf, 2005.

[35] Dash, M., and H. Liu, Feature Selection for Classification. Intelligent Data Analysis, 1997: p. $131 \mathrm{C} 156$.

[36] Devi, K.N., V.M. Bhaskaran and G.P. Kumar, Cuck

o ,Optimized SVM for Stock Market Prediction, IEEE Sponsored 2nd International Conference on Innovations in Information, Embedded, and Communication Systems (ICJJECS)2015, 2015.

[37] Dai, W., Y.E. Shao, and C. Lu, Incorporating feature selection method into support vector regression for stock index forecasting. Neural Computing and Applications, 2013. 23(6): p. 15511561.

[38] Yanan Mao, Zuoquan Zhang, Dingyuan Fan, "Hybrid feature selection based on improved genetic algorithm for stock prediction," 2016 6th International Conference on Digital Home,2016.

[39] Dina A. Ragab, Maha Sharks and Omneya Attallah," Breast Cancer Diagnosis Using an Efficient CAD System Based on Multiple Classifiers," Diagnostics 2019, 9, 165; DOI:10.3390/diagnostics9040165.

[40] David P Salmon and Mark W. Bondi“Neuropsychological Assessment of Dementia" Access NIH public, PubMed central, US national library of medicine Institutes of Health, May 2010.

[41] Divya Jain, Vijendra Singh," Feature selection and classification systems for chronic disease prediction: A review," Egyptian Informatics Journal 19 (2018) 179-189,2018

[42] Ramon Casanova, Fang-Chi Hsu, Kaycee M Sink, Stephen R Rapp, Je_ D Williamson, Susan M Resnick, Mark A Espeland, Alzheimer's Disease Neuroimaging Initiative, et al. Alzheimer's disease risk assessment using large-scale machine learning methods. PloS one, 8(11):e77949, 2013. 
[43] Susanne G Mueller, Michael W Weiner, Leon J Thal, Ronald C Petersen, Cli_ord Jack, William Jagust, John Q Trojanowski, Arthur W Toga, and Laurel Beckett. The Alzheimer's disease neuroimaging initiative. Neuroimaging Clinics of America, 15(4):869\{877, 2005.

[44] Elizabeth G Kehoe, Jonathan P McNulty, Paul G Mullins, and Arun LW Bode.Advances in MRI Biomarkers for the Diagnose of AD. Biomarkers in Medicine, 8(9):1151\{1169, 2014.

[45] Arthur W Toga and Karen L Crawford. The AD Neuroimaging informatics core: A decade in review. Alzheimer's and Dementia: The Journal of the Alzheimer's Association, 11(7):832\{839, 2015.

[46] Michael W Weiner, Dallas P Veitch, Paul S Aisen, Laurel A Beckett, Nigel J Cairns, Jesse Cedarbaum, Robert C Green, Danielle Harvey, Cli_ord R Jack, William Jagust, et al. 2014 Update of the AD Neuroimaging: A review of papers published since its inception. Alzheimer's and Dementia: The Journal of the Alzheimer's Association, 11(6):e1 \{e120, 2015.

[47]A benchmark Alzheimer's MRI segmentation Dataset from online https://www.kaggle.com/tourist55/alzheimers-dataset-4-class-of-images (accessed online $12 / 05 / 2019)$.

[48]N. Woods, O. Longe, and A. Roberts, "A Sobel edge detection algorithm based system for analyzing and classifying image-based spam," Journal of Emerging Trends in Computing and Information Sciences, vol. 3, no. 4, 2012.View at: Google Scholar.

[49] G.Saianilkumar1, K.Ravindrakbabu2, B.V. Nagendra Prasad Reddy3, "Medical Image Retrieval Based On Edge Histogram Descriptor," International Journal of Engineering Science Invention ISSN (Online): 2319 - 6734, ISSN (Print): 2319 - 6726 www.ijesi.org ||Volume 4 Issue 4 || April 2015 || PP.22-27.

[50] Jianfang Cao," Implementing a Parallel Image Edge Detection Algorithm Based on the OtsuCanny Operator on the Hadoop Platform, "Computational Intelligence and Neuroscience, Volume 2018 |Article ID 3598284 | 12 pages | https://doi.org/10.1155/2018/3598284.

[51] Zhu N, Zhang D, Wang W, et al. A Novel Coronavirus from Patients with Pneumonia in China, 2019[J]. N Engl J Med. 2020 Jan 24. DOI:10.1056/NEJMoa2001017.

[52]Li Q, Guan X, Wu P, et al. Early Transmission Dynamics in Wuhan, China, of Novel Coronavirus-Infected Pneumonia[J]. N Engl J Med. 2020 Jan 29. DOI:10.1056/NEJMoa2001316.

[53] Cohen J, Normile D. New SARS-like virus in China triggers alarm[J]. Science.2020 Jan 17; 367(6475):234-235. DOI: 10.1126/science.367.6475.234.

[54]Corman VM, Landt O, Kaiser M, et al. Detection of 2019 novel coronavirus(2019-nCoV) by real-time RT-PCR[J]. Euro Surveill. 2020 Jan; 25(3). DOI:

10.2807/1560-7917.ES.2020.25.3.2000045.[55]Huang C, Wang Y, Li X, et al. Clinical features of patients infected with 2019novel coronavirus in Wuhan, China[J]. Lancet. 2020 Jan 24. PII: S01406736(20)30183-5. DOI:10.1016/S0140-6736(20)30183-5.

[56]Chan JF, Yuan S, Kok KH, et al. A familial cluster of pneumonia associated with the 2019 novel coronavirus indicating person-to-person transmission: a study of a family cluster[J]. Lancet. 2020 Jan 24. PII: S0140 6736(20)30154-9. DOI:10.1016/S0140-6736(20)30154-9.

[57]Liu X, Guo S, Yang B, et al. Automatic Organ Segmentation for CT Scans Based on Super-Pixel and Convolutional Neural Networks[J]. Journal of Digital Imaging,2018, 31(6).26 / 29

[58]Gharbi, Michaël, Chen J, Barron J T, et al. Deep Bilateral Learning for Real-Time Image Enhancement[J]. Acm Transactions on Graphics, 2017, 36(4):118.

[59]Hesamian M H, Jia W, He X, et al. Deep Learning Techniques for Medical Image Segmentation: Achievements and Challenges[J]. Journal of Digital Imaging, 2019,32(8).

[59]Akagi M, Nakamura Y, Higaki T, et al. Correction to Deep learning reconstruction improves the image quality of abdominal ultra-high-resolution CT[J].European Radiology, 2019, 29(8).

[60] Nardelli P, Jimenez-Carretero D, Bermejo-Pelaez D, et al. Pulmonary Artery-Vein Classification in CT Images Using Deep Learning[J]. IEEE Transactions on Medical Imaging, 2018, PP(99):1-1.

[61]Zhu W, Huang Y, Zeng L, et al. AnatomyNet: Deep learning for fast and fully automated wholevolume segmentation of head and neck anatomy[J]. medical physics, 2019, 46(2).

[62]Huang P, Park S, Yan R, et al. Added Value of Computer-aided CT Image Features for Early Lung Cancer Diagnosis with Small Pulmonary Nodules: A Matched Case-Control Study. Radiology. 2018 Jan; 286(1):286-295. DOI:1148/radiol.2017162725. 
[63] Ardila D, Kiraly AP, Bharadwaj S, et al. End-to-end lung cancer screening with deep threedimensional learning on low-dose chest computed tomography. Nat Med. 2019 Jun;25(6):954-961. DOI: 10.1038/s41591-019-0447-x.

[65]Esteva A, Kuprel B, Novoa RA, et al. Dermatologist-level classification of skin 27 / 29cancer with deep neural networks. Nature. 2017 Feb 2;542(7639):115-118. DOI:101038/nature21056. Epub 2017 Jan 25.

[66] Lakhani P, Sundaram B. Deep Learning at Chest Radiography: Automated Classification of Pulmonary Tuberculosis by Using Convolutional Neural Networks[J]. Radiology, 2017:162326.

[67]Wu W, Li X, Du P, et al. A Deep Learning System That Generates Quantitative CT Reports for Diagnosing Pulmonary Tuberculosis. arXiv preprint arXiv:1910.02285, 2019.

[68]Li L, Huang H, Jin X. AE-CNN Classification of Pulmonary Tuberculosis Based on CT Images[C]// 2018 9th International Conference on Information Technology in Medicine and Education (ITME). IEEE Computer Society, 2018.

[69]Onisko A, Druzdzel M, Wasyluk H. Learning Bayesian network parameters from small data sets: application of Noisy-OR gates[J]. International Journal of Approximate Reasoning, 2001, 27(2):165182.

[70]Milletari F, Navab N, Ahmadi S A. V-Net: Fully Convolutional Neural Networks for Volumetric Medical Image Segmentation. arXiv preprint arXiv:1606.04797,2016.

[71]Jeffrey P Kanne. Chest CT Findings in 2019 Novel Coronavirus (2019-nCoV) Infections from Wuhan, China: Key Points for the Radiologist[J].Radiology, 2020.

[72]Chung M, Bernheim A, Mei X, et al. CT Imaging Features of 2019 Novel Coronavirus (2019nCoV) [J]. Radiology, 2020.

[73] He K, Zhang X, Ren S, et al. Deep Residual Learning for Image Recognition[J].28 / 292015.

[74]Gamal M. I.Selim Dr Hatem Khater, A. A. F. (2020). A Composite Hybrid Feature Selection Learning-Based Optimization of Genetic Algorithm For Breast Cancer Detection. 2nd International Conference on Advanced Research in Applied Science and Engineering, Which Will Be Held in Oxford, UK.

[75]Gamal M. I.Selim Dr Hatem Khater, E. A. A. F. (2020). Applying Artificial Intelligence Techniques to Improve Clinical Diagnosis of Alzheimer's Disease. The 9th International Conference on Research in Science and Technology (RSTCONF), 20-22 March 2020 in Berlin, Germany .

[76]Gamal M. I.Selim Dr Hatem Khater, E. A. A. F. (2020). A Novel Approach of CT Images Feature Analysis and Prediction to Screen for Corona Virus Disease (COVID-19). (International Journal of Scientific and Engineering Research (IJSER) - (ISSN 2229-5518, 11(3), 9.

[77]Gamal M. I.Selim Dr Hatem Khater, E. A. A. F. (2020). Applying Artificial Intelligence Techniques for Prediction of Neurodegenerative Disorders: A Comparative Case-Study on Clinical Tests and Neuroimaging Tests with Alzheimer's Disease. 2nd International Conference on Advanced Research in Applied Science and Engineering, Which Will Be Held in Oxford, UK. 